\title{
Citometría de flujo en reticulocitos de sangre periférica como indicador de inestabilidad cromosómica en pacientes con gliomas de alto grado
}

\author{
Lina Marcela Barrera', León Darío Ortiz ${ }^{1,2}$, Hugo Grisales ${ }^{3}$, Mauricio Rojas ${ }^{4}$, Mauricio Camargo ${ }^{1}$ \\ 1 Grupo de Genética, Regeneración y Cáncer (GRC), Instituto de Biología, Universidad de Antioquia, Medellín, \\ Colombia \\ 2 Instituto de Cancerología-Clínica Las Américas, Medellín, Colombia \\ 3 Grupo de Investigación Demografía y Salud, Facultad Nacional de Salud Pública, Universidad de Antioquia, \\ Medellín, Colombia \\ 4 Unidad de Citometría de Flujo y Grupo de Inmunología Celular e Inmunogenética (GICIG), Facultad de Medicina, \\ Universidad de Antioquia, Medellín, Colombia
}

Introducción. La cuantificación de la inestabilidad cromosómica es un parámetro importante para evaluar la genotoxicidad y la radiosensibilidad. Las técnicas convencionales requieren cultivos celulares - laboriosos análisis microscópicos de cromosomas o núcleos. La citometría de flujo en reticulocitos ha surgido como una alternativa para los estudios in vivo, ya que reduce los tiempos de análisis e incrementa hasta en 20 veces el número de células analizables.

Objetivos. Estandarizar los parámetros de citometría de flujo requeridos para seleccionar y cuantificar reticulocitos micronucleados (RET-MN) a partir de muestras de sangre periférica, y cuantificar la frecuencia de esta subpoblación anormal como medida de inestabilidad citogenética en sendas poblaciones de voluntarios sanos $(n=25)$ y pacientes $(n=25)$ recién diagnosticados con gliomas de alto grado antes de iniciar el tratamiento.

Materiales y métodos. Las células sanguíneas se marcaron con anti-CD71-PE para reticulocitos, antiCD61-FITC para la exclusión de plaquetas y yoduro de propidio para detectar el ADN en reticulocitos. La fracción celular MN-RET ${ }^{\mathrm{CD} 71+}$ se seleccionó y se cuantificó con un citómetro de flujo automático.

Resultados. Se describió detalladamente la estandarización de los parámetros citométricos, con énfasis en la selección y la cuantificación de la subpoblación celular MN-RETCD71+. Se establecieron los niveles basales de MN-RET ${ }^{C D 71+}$ en la población de control y en los pacientes se encontró un incremento de 5,2 veces antes de iniciar el tratamiento $(p<0,05)$.

Conclusión. Los resultados evidenciaron la utilidad de la citometría de flujo acoplada a la marcación de las células $\mathrm{RET}^{\mathrm{CD} 71+}$ como método eficiente para cuantificar la inestabilidad cromosómica in vivo. Se sugieren posibles razones del incremento de micronúcleos en células $\mathrm{RET}^{\mathrm{CD} 71+}$ de pacientes con gliomas.

Palabras clave: citometría de flujo; reticulocitos; inestabilidad cromosómica; glioma; pruebas de micronúcleos.

doi: https://doi.org/10.7705/biomedica.v38i4.3882

Flow cytometry in peripheral blood reticulocytes as a marker of chromosome instability in highgrade glioma patients

Introduction: The quantification of chromosomal instability is an important parameter to assess genotoxicity and radiosensitivity. Most conventional techniques require cell cultures or laborious microscopic analyses of chromosomes or nuclei. However, a flow cytometry that selects the reticulocytes has been developed as an alternative for in vivo studies, which expedites the analytical procedures and increases up to 20 times the number of target cells to be analyzed.

Objectives: To standardize the flow cytometry parameters for selecting and quantifying the micronucleated reticulocytes ${ }^{\mathrm{CD71+}}(\mathrm{MN}-\mathrm{RET})$ from freshly drawn peripheral blood and to quantify the

\section{Contribución de los autores:}

Lina Marcela Barrera: toma de muestras, estandarización de la técnica, ensayos experimentales, análisis estadísticos y análisis de resultados

León Darío Ortiz: diseño de la logística para el estudio clínico de los pacientes

Hugo Grisales: análisis estadísticos

Mauricio Rojas: análisis de los datos y resultados de la citometría de flujo

Mauricio Camargo: concepción y diseño del estudio, supervisión del desarrollo del trabajo y análisis de los datos

Todos los autores participaron en la escritura del manuscrito. 
frequency of this abnormal cell subpopulation as a measure of cytogenetic instability in populations of healthy volunteers $(n=25)$, and patients $(n=25)$, recently diagnosed with high-grade gliomas before the onset of treatment.

Materials and methods: Blood cells were methanol-fixed and labeled with anti-CD-71-PE for reticulocytes, antiCD-61-FITC for platelet exclusion, and propidium iodide for DNA detection in reticulocytes. The $\mathrm{MN}-\mathrm{RET}^{\mathrm{CD} 71+}$ cell fraction was selected and quantified with an automatic flow cytometer. Results: The standardization of cytometry parameters was described in detail, emphasizing the selection and quantification of the MN-RET ${ }^{\mathrm{CD} 71+}$ cellular fraction. The micronuclei basal level was established in healthy controls. In patients, a 5.2-fold increase before the onset of treatment was observed $(p<0.05)$. Conclusion: The data showed the usefulness of flow cytometry coupled with anti-CD-71-PE and antiCD-61-FITC labeling in circulating reticulocytes as an efficient and high resolution method to quantify chromosome instability in vivo. Finally, possible reasons for the higher average of micronuclei in $\mathrm{RET}^{\mathrm{CD} 71+}$ cells from untreated high-grade glioma patients were discussed.

Key words: Flow cytometry; reticulocytes; chromosome instability; glioma; micronucleus tests. doi: https://doi.org/10.7705/biomedica.v38i4.3882

La cuantificación de la inestabilidad cromosómica es un parámetro importante para estimar la radiosensibilidad y evaluar la genotoxicidad relacionada con la exposición terapéutica, ocupacional, ambiental o accidental. Los métodos convencionales emplean la visualización microscópica de daños cromosómicos o nucleares, sin precultivo celular o con él, como es el caso del ensayo clásico de alteraciones cromosómicas en linfocitos en división, o el de micronúcleos en linfocitos binucleados (1-5). Su amplio uso y su validez son incuestionables, pero demandan laboriosos análisis visuales de, por lo menos, un centenar de mitosis o dos mil células interfásicas por muestra, lo cual dificulta la rápida obtención de resultados. De ahí que la automatización o el empleo de la citometría de flujo constituyan alternativas para acortar los tiempos de análisis $(3,6,7)$, y aumentar el poder estadístico, incrementando hasta en 20 veces el número de células analizables por muestra, como se evidencia en el presente reporte.

Los eritroblastos son una población celular mitóticamente activa, precursora de eritropoyesis, con nicho en la médula ósea, cuyos núcleos se expulsan pocas horas después de la última mitosis. De allí se generan células enucleadas denominadas eritrocitos inmaduros o reticulocitos. Estas células recién producidas en la médula ósea fueron el blanco de observación para la prueba original in vivo de micronúcleos en extendidos de médula

\section{Correspondencia:}

Mauricio Camargo, Sede de Investigación Universitaria, Universidad de Antioquia, Carrera $53 \mathrm{~N}^{\circ}$ 61-30, torre 2, laboratorio 432, Medellín, Colombia

Teléfono: (574) 2196528

mauricio.camargo@udea.edu.co

Recibido: 25/04/17; aceptado: 15/03/18 ósea de ratones estandarizado por Schmid $(8,9)$. En humanos, los reticulocitos constituyen una fracción minoritaria de los eritrocitos circulantes, lo cual dificulta su empleo en esta prueba mediante microscopía. La citometría de flujo, en cambio, facilita su detección mediante la marcación diferencial de los reticulocitos con el anticuerpo anti-CD-71, el cual detecta la transferrina, que es la proteína encargada de transportar el hierro y es específica para esta población. El tamaño de los reticulocitos es similar al de las plaquetas, las cuales se pueden diferenciar, filtrar y descartar, empleando anti-CD-61 y citometría de flujo (10).

Por el otro lado, la evaluación de la genotoxicidad a nivel cromosómico en linfocitos de sangre periférica, se ha hecho tradicionalmente en cultivos celulares con presencia de un mitógeno, en tanto que el ensayo de micronúcleos basado en los reticulocitos, no tiene este requisito; además, una vez estandarizada la metodología, las células obtenidas no necesitan el precultivo y pueden procesarse de inmediato siguiendo procedimientos mínimos (3). Este es un método práctico y expedito para evaluar in vivo la inestabilidad cromosómica constitutiva o del daño genómico inducido, en personas expuestas a genotóxicos potenciales por razones terapéuticas, ocupacionales, ambientales o accidentales. Eventualmente, también se podría utilizar para evaluar la radiosensibilidad en pacientes que deban someterse a radioterapia.

El presente estudio se diseñó con el fin de estandarizar y adaptar la metodología propuesta por Dertinger, et al. (11), para cuantificar micronúcleos en células RET $\mathrm{RD}^{\mathrm{C} 71+}$ mediante citometría de flujo de tres canales, establecer el número basal de reticulocitos micronucleados en una población de voluntarios sanos, y cuantificar la frecuencia in 
vivo de micronúcleos en sangre periférica, en una población de pacientes con gliomas de alto grado, como forma de medir la inestabilidad citogenética antes del tratamiento.

\section{Materiales y métodos}

\section{Reactivos}

Se emplearon metanol absoluto (Merck, USA, 15680090), heparina sódica (Grand Pharma), solución de sales de Hank (HBSS-H1387), RNAsa (R5000) y el yoduro de propidio (P4170) de Sigma (St Louis, MO, USA), así como los anticuerpos humanos anti-CD71 (clon T56/14) y anti-CD61 (clon F11) de Invitrogen ${ }^{\mathrm{TM}}$ (Camarillo, CA, USA).

\section{Población de estudio}

Se incluyeron 50 voluntarios en el estudio: 25 personas sanas que fueron reclutadas en la Universidad de Antioquia y 25 pacientes recién diagnosticados con glioma de alto grado en el Instituto de Cancerología de la Clínica Las Américas en un lapso de 12 meses. A cada individuo se le tomó una muestra de $4 \mathrm{ml}$ de sangre venosa en un tubo Vacutainer ${ }^{\mathrm{TM}}$ con heparina.

Los consentimientos informados fueron firmados por los voluntarios sanos antes de la toma de la muestra y por los pacientes después del diagnóstico y previa explicación por parte del médico. Los pacientes fueron pareados por edad ( \pm 5 años) y sexo.

\section{Fijación de la muestra}

Se agregó $1 \mathrm{ml}$ de sangre tratada con heparina a un tubo cónico con $5 \mathrm{ml}$ de diluyente $(500$ unidades de heparina/ml: Hanks's Balanced Salt Solution, HBSS); luego se fijó la muestra adicionando $450 \mu \mathrm{l}$ de la dilución anterior en $5 \mathrm{ml}$ de metanol frío $\left(-80^{\circ} \mathrm{C}\right)$, y se agitó en un agitador de vórtice durante 20 segundos, para luego almacenarla a $-80{ }^{\circ} \mathrm{C}$ durante 24 horas.

\section{Marcación con yoduro de propidio y anticuerpos conjugados}

Después de la fijación, $1 \mathrm{ml}$ de la muestra se agregó a un tubo cónico con $13 \mathrm{ml}$ de diluyente HBSS, se centrifugó durante cinco minutos a $600 \mathrm{~g}$ y se descartó el sobrenadante por decantación; luego se agitó brevemente para resuspender el botón celular y se adicionaron $20 \mu \mathrm{l}$ de esta suspensión de células a un tubo de citometría (polipropileno, $12 \times 75 \mathrm{~mm}$ ) que contenía $75 \mu \mathrm{l}$ de solución ARNsa $(980 \mu \mathrm{l}$ de HBSS y $20 \mu \mathrm{l}$ de ARNsa $1 \mathrm{mg} / \mathrm{ml})$.
Las muestras se analizaron usando tres marcadores: antiCD-71-PE, para identificar reticulocitos, anti-CD-61-FITC, para la exclusión citométrica de las plaquetas, y yoduro de propidio, para cuantificar la cantidad relativa de ADN. Se agregaron $5 \mu \mathrm{l}$ de cada anticuerpo siguiendo las recomendaciones del proveedor.

Las muestras se incubaron consecutivamente durante 30 minutos a $4{ }^{\circ} \mathrm{C}$ y, luego durante 60 minutos a temperatura ambiente. Pasado este tiempo, se lavaron con $3 \mathrm{ml}$ de HBSS y se centrifugaron durante cinco minutos a $600 \mathrm{~g}$; luego, se adicionó $1 \mathrm{ml}$ de solución de yoduro de propidio (PI) $(1,25 \mu \mathrm{g} / \mathrm{m}$ de $\mathrm{PI}$ en HBSS). Por último, se almacenaron durante 15 minutos a $4{ }^{\circ} \mathrm{C}$ y se leyeron en un citómetro de flujo FACS Canto II ${ }^{\mathrm{TM}}$ (BD-Immunocytometry System, San José, CA).

Las células fueron excitadas con un láser de 488 $\mathrm{nm}$ y se analizó la emisión de los fluorocromos a 530/30 nm (FITC), 585/42 (PE) nm y 670/LP nm (yoduro de propidio). La adquisición de eventos para cada muestra procedió hasta que el número de células RET ${ }^{\mathrm{CD} 71+}$ fue mayor o igual a 80.000 o hasta que se agotó el volumen de la muestra. El porcentaje de células $\mathrm{RET}^{\mathrm{CD} 71+\mathrm{Pl}+}$ se determinó en cada muestra de sangre.

\section{Cálculo de la compensación}

Para calcular la matriz de compensación, se ajustaron los voltajes de tamaño, granularidad y fluorescencia de las muestras con una muestra de células sin tinción. Sobre dichos voltajes, se calculó la matriz de compensación utilizando las micropartículas Compbeads revestidas con anticuerpos de captura anti-Mouse $\lg _{\mathrm{k}}$ mezcladas con cantidades equivalentes de perlas de control negativo.

El control de compensación para el yoduro de propidio se construyó utilizando el control negativo de las micropartículas CompBeats mezcladas con núcleos de eritrocitos de pollo (BD ${ }^{\mathrm{TM}} \mathrm{CEN}$ ).

La matriz se calculó de forma automática en el programa BD FACSDivaTM, versión 6.1.3. La estabilidad en los índices de tinción se verificó en, por lo menos, tres momentos independientes. La estabilidad en los voltajes y la eficiencia de los detectores se verificaron mediante esferas BD Cytometer Setup and Tracking ${ }^{\mathrm{TM}}$ (CST). Los parámetros de lectura estuvieron dentro de las variantes aceptadas para el instrumento, estimados mediante las gráficas de Levey-Jennings construidas a partir de la señal de los detectores implicados en el análisis de los micronúcleos. 


\section{Estándares biológicos para la verificación de la compensación}

En los análisis de citometría, se verificó la corrección del derramamiento espectral entre los fluorocromos mediante tinciones individuales para cada marcador de superficie según lo descrito por Dertinger, et al., y Torous, et al. (12-14). Esta misma estrategia permitió establecer la positividad de las células en cada región.

Se prepararon cuatro muestras de cada individuo. La primera contenía $20 \mu \mathrm{l}$ de la muestra con yoduro de propidio, la segunda, anti-CD-71-PE, y la tercera, anti-CD-61-FITC. Con estas se hizo la corrección de la compensación y con el cuarto tubo, que contenía ambos anticuerpos, se analizó el porcentaje de células CD61-CD71+ PI+ después de haber excluido células $2 n$ y los agregados mediante una región definida sobre FSC-A Vs. FSC-H; luego, se determinó la frecuencia de reticulocitos micronucleados en la fracción de células $\mathrm{RET}^{\mathrm{CD} 71+}$.

\section{Adquisición de datos y análisis estadísticos}

Para la estimación de las frecuencias de reticulocitos micronucleados, se utilizó el programa de citometría de flujo FlowJoTM 7.6.2.

En cuanto al análisis estadístico univariado, se acudió a los indicadores clásicos de resumen, a saber, media, mediana, desviación estándar y coeficiente de variación para las variables cuantitativas, y porcentajes para las variables cualitativas.

En el análisis bivariado, dada la no normalidad del resultado en su escala original (establecida con la prueba de Shapiro-Wilk), se utilizó la prueba no paramétrica $U$ de Mann-Whitney para determinar la diferencia en los rangos promedios del porcentaje de reticulocitos micronucleados con cada uno de los factores que ameritaron valoración.

En el análisis multivariado, se construyó un modelo de regresión logística explicativo, tomando como variable dependiente el porcentaje de dichos reticulocitos (normal-alto).

Debe advertirse que la categorización se originó al segmentar la variable original con un nivel de medición de razón y el porcentaje de reticulocitos micronucleados utilizando la media como criterio estadístico.

Se consideraron como variables independientes aquellas establecidas como importantes por su efecto sobre el resultado. La finalidad en la construcción del modelo de regresión logística estribó en controlar el efecto presuntamente confusor de cada una de las variables incluidas en el modelo frente a las otras. Estos resultados estadísticos se obtuvieron utilizando el programa SPSS ${ }^{\mathrm{TM}}$, versión 22.

\section{Consideraciones éticas}

Además de la firma del consentimiento informado, se obtuvo la aprobación del Comité de Ética Independiente del Instituto de Cancerología de la Clínica Las Américas y del Comité de Bioética de la Sede de Investigación de la Universidad de Antioquia.

\section{Resultados}

\section{Selección de la población de análisis}

Inicialmente, se descartaron los eventos celulares mayores a $2 n$ mediante los parámetros de exclusión FSC-H y la incorporación de yoduro de propidio (figura 1a); de allí, se tomaron los eventos menores de $2 \mathrm{n}(\mathrm{P} 1)$ y se excluyeron los agregados (P2) mediante el uso de los parámetros FSC-H y FSC-A (figura 1b). En la figura 1c se muestra la distribución de las poblaciones celulares en cuanto a su tamaño (FSC-A) y granularidad (SSC-A). Posteriormente, y dado que las plaquetas tienen un tamaño similar a la porción de reticulocitos inmaduros, se utilizó el anticuerpo anti-CD61 para excluirlas (figura 1d), y se seleccionó la población correspondiente a células $\mathrm{RET}^{\mathrm{CD} 71+}$ (figura 1e). Por último, en la población seleccionada, se cuantificaron los eventos con mayor cantidad de yoduro de propidio, es decir, el cuadrante Q2-2 correspondiente a los reticulocitos micronucleados (figura 1f).

\section{Medición de la población de reticulocitos micro- nucleados}

Las muestras de los 50 individuos (25 pacientes y 25 controles) se analizaron para detectar las frecuencias de células $\mathrm{RET}^{\mathrm{CD} 71+}$ y de reticulocitos micronucleados. El número de eventos únicos totales evaluados por individuo osciló entre 28.000 y 520.000 , de los cuales alrededor del $1 \%$ pertenecía a la población total de reticulocitos. Con base en el valor promedio de células RETCD71+ para estos individuos, y en los análisis con los cuales se midieron las frecuencias de reticulocitos establecidos sobre la fluorescencia asociada a los ácidos nucleicos, se estimó que el anticuerpo anti-CD-71-PE había marcado, aproximadamente, entre 98 y $100 \%$ de la población de reticulocitos.

\section{Comparación de las frecuencias de RET-MN de pacientes y controles}

Inicialmente, se analizó el porcentaje de reticulocitos micronucleados en las muestras de voluntarios 
sanos, el cual varió entre 0,00 y $8,48 \%$, con una mediana de $2,31 \%$ y un rango intercuartil de 3,51 . En los pacientes con cáncer (gliomas de alto grado), por el contrario, las frecuencias de estos reticulocitos fueron mayores que en los controles $(0,384 ; 61,76 \%)$, con una mediana de $12,45 \%$ y un rango intercuartílico de 18,325. Los datos de frecuencias se presentan en el cuadro 1.

En la figura 2 se presenta una gráfica bivariada que compara la frecuencia de las $\mathrm{RET}^{\mathrm{CD} 71+}$ de un voluntario sano (control) (figura 2a) y la de un paciente con glioma de alto grado antes del tratamiento (figura 2b); como se observa en el cuadrante Q2-3, el número de reticulocitos micronucleados fue mucho más elevado en el paciente que en el control.

En el análisis multivariado solo los casos tuvieron un efecto significativo sobre el porcentaje de reticulocitos micronucleados; de hecho, su porcentaje de riesgo alto (por encima de la mediana) fue 93,9\% más bajo en los controles que en los casos después de ajustar por las variables de ser hombre, consumir alcohol o tabaco, no consumir antioxidantes, usar medicamentos y ser adulto mayor (cuadro 2). a.

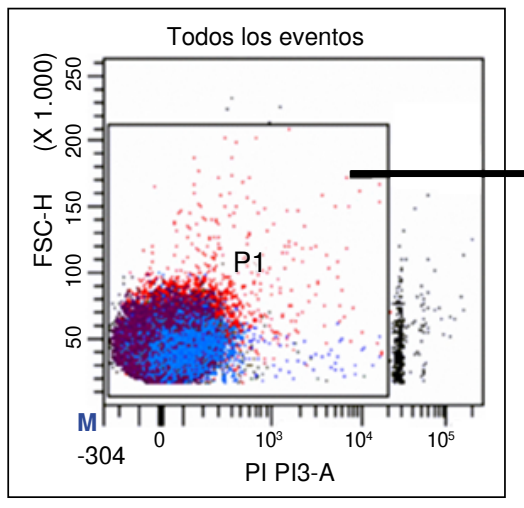

d.

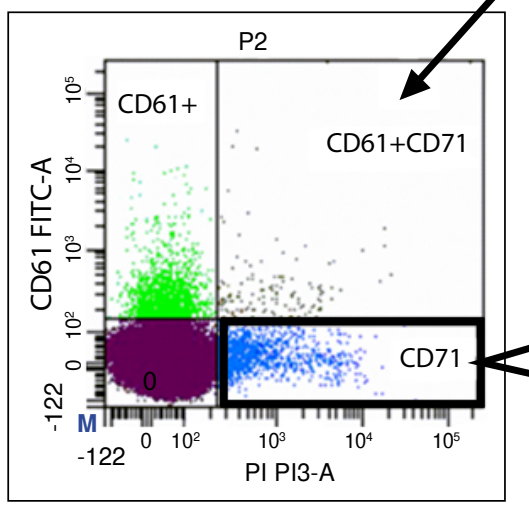

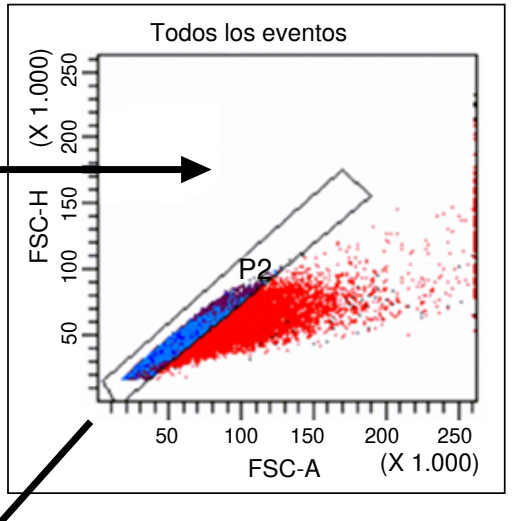

e.

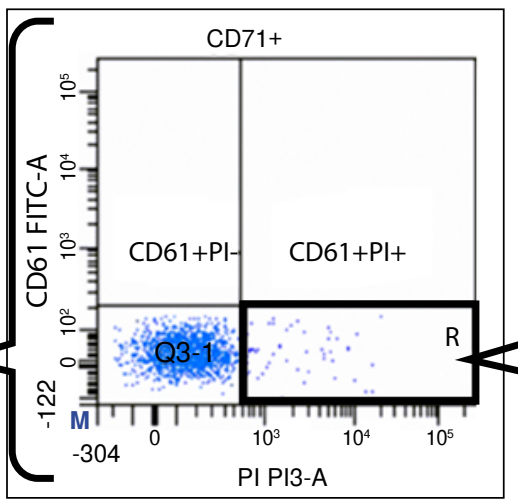

C.

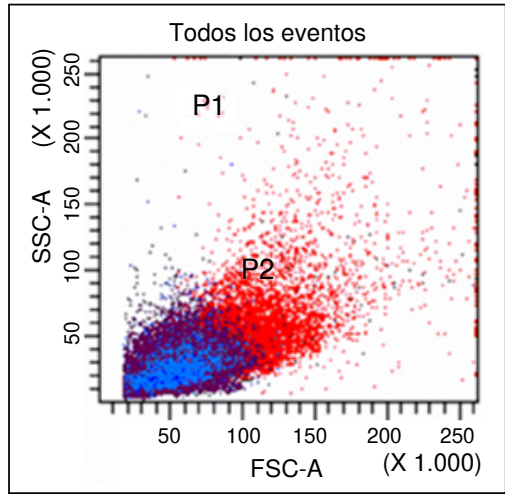

f.

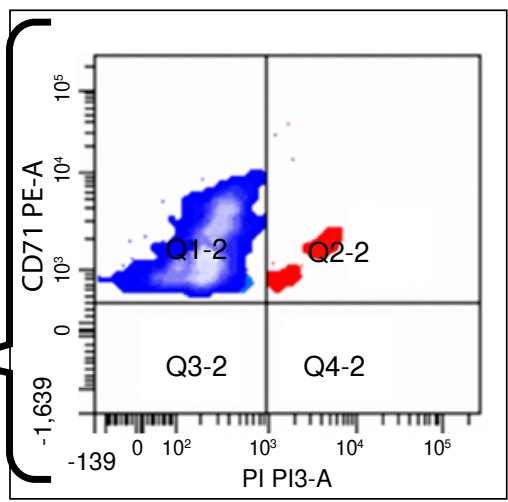

Figura 1. Gráficas bivaridas que ilustran la estrategia de portales (gating) usada para los análisis de muestras de sangre humana. a) Selección de eventos (<2n, P1); sobre el límite superior de esta región se observan las células 2n. b) Exclusión de agregados utlizando los parámetros de FSC-Area Vs. FSC-Height. c) Distribución de tamaño (FSC-A) Vs. granularidad (SSC-A); en color rojo aparecen los agregados y en azul los eventos únicos. d) Exclusión de plaquetas CD61+. e) Selección de la población celular $\mathrm{RET}^{\mathrm{CD71+}}$. f) Cuantificación de reticulocitos multinucleados (RET-MN) (CD71+PI+)

Cuadro 1. Medidas estadísticas descriptivas del porcentaje de reticulocitos micronucleados.

\begin{tabular}{lcccccc}
\hline Porcentaje inicial de RET-MN & $\mathbf{n}$ & Media & Mediana & Desviación estándar & CV & $\mathbf{p}$ \\
\hline Casos & 25 & 12,45 & 3,45 & 16,74 & 134 & 0,045 \\
Controles & 25 & 2,97 & 2,31 & 2,36 & 79,46 & 0,045 \\
\hline
\end{tabular}

RET-MN: reticulocitos micronucleados; CV: coeficiente de variación

Los valores de $p<0,05$ indican diferencias significativas entre controles y pacientes. 
Aunque las demás variables constitutivas del modelo no tuvieron significación estadística, cabe mencionar que, al ajustar por las variables mencionadas, el riesgo de un porcentaje alto de reticulocitos micronucleados fue $68 \%$ mayor en los hombres que en las mujeres, y 5,3\% mayor en adultos mayores que en los adultos jóvenes.

Por último, el análisis de las variables independientes evidenció que estas solo explicaron el $42,3 \%$ de la presencia de reticulocitos micronucleados.

\section{Discusión}

La detección de micronúcleos por citometría de flujo en reticulocitos humanos se basa en la marcación de la población de interés y la cuantificación de dichos micronúcleos en estas células de manera específica, siguiendo un protocolo de fijación y marcación celular diferencial en muestras de sangre periférica.

Los procedimientos descritos en este artículo son sencillos y se basan en el uso de colorantes de ácidos nucleicos con gran especificidad para el ADN, así como los reportados por Dertinger, et al.

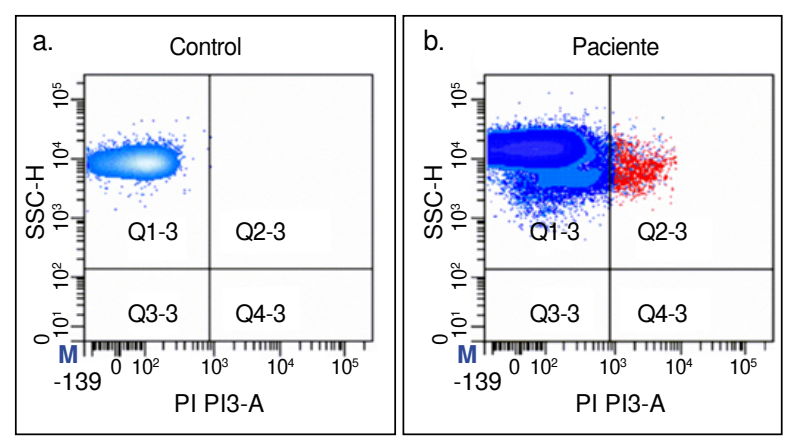

Figura 2. Gráfica bivariada que ilustra la resolución de fluorescencia de la subpoblación celular $\mathrm{RET}^{\mathrm{CD} 71+}$ : a) en sangre periférica de un voluntario sano, y b) en un paciente con glioma antes de iniciar el tratamiento.
La técnica no incluye la separación física de los eritrocitos CD71+ sino el uso de anticuerpos contra CD71 marcados con fluoróforos, que se utilizan para distinguir la fracción inmadura de los eritrocitos en el citómetro de flujo $(6,12,13)$.

La eficiencia con la cual el bazo humano remueve de la circulación los eritrocitos que contienen micronúcleos, se ha considerado un obstáculo para utilizar la medida de reticulocitos micronucleados en sangre periférica como un indicador confiable del daño citogenético $(12,13)$. Sin embargo, los datos presentados en este estudio sugieren que sería posible evaluar el daño citogenético en individuos no sometidos a esplenectomía utilizando un sistema de análisis de alto rendimiento, como el de la citometría de flujo, con el cual se puede detectar la rara aparición de la subpoblación $\mathrm{RET}^{\mathrm{CD} 71+}$ y de los micronúcleos.

Con base en los resultados obtenidos y la efectividad de este procedimiento, se puede afirmar que los reticulocitos recién formados en sangre periférica representan un blanco celular alternativo con muchas ventajas: se requieren bajos volúmenes sanguíneos; son muy pocos los pasos de procesamiento de la muestra, pues la cosecha de células es mínimamente invasiva; los requisitos del equipo son poco sofisticados, y el conteo es automatizado e imparcial, pues el análisis de muestras de sangre para la detección de micronúcleos excede las tasas de hasta 1.000 eventos por segundo, lo cual equivale a un gran número de células si se compara con las empleadas en microscopia óptica, en la cual se analizan máximo 2.000 células binucleadas por muestra (14). Además, se cuantifica simultáneamente el porcentaje de reticulocitos y el de reticulocitos micronucleados, y el tiempo requerido para recolectar los datos en el citómetro es de 20 minutos por muestra, aproximadamente (13).

Cuadro 2. Regresión logística del análisis multivariado para el porcentaje del riesgo de reticulocitos micronucleados (MN-RET) según los factores de interés

\begin{tabular}{|c|c|c|c|c|c|c|c|}
\hline \multirow[t]{2}{*}{ Factores } & \multirow[t]{2}{*}{ Coeficiente } & \multirow{2}{*}{$\begin{array}{c}\text { Error } \\
\text { estándar }\end{array}$} & \multirow{2}{*}{$\begin{array}{l}\text { Prueba } \\
\text { de Wald }\end{array}$} & \multirow{2}{*}{$\begin{array}{l}\text { Valor } \\
\text { de } p\end{array}$} & \multirow{2}{*}{$\begin{array}{l}\text { Odds } \\
\text { ratio }\end{array}$} & \multicolumn{2}{|c|}{ IC $_{95 \%}$ para OR } \\
\hline & & & & & & Inferior & Superior \\
\hline Caso & $-2,80$ & 1,25 & 5,04 & 0,025 & 0,06 & 0,005 & 0,70 \\
\hline Ser hombre & 0,52 & 0,93 & 0,32 & 0,575 & 1,69 & 0,270 & 10,50 \\
\hline Consumo elevado de alcohol & $-0,11$ & 0,35 & 0,09 & 0,760 & 0,90 & 0,460 & 1,78 \\
\hline Consumo elevado de cigarrillo & $-0,11$ & 0,53 & 0,04 & 0,839 & 0,90 & 0,320 & 2,52 \\
\hline No consumir antioxidantes & $-1,02$ & 0,99 & 1,07 & 0,302 & 0,36 & 0,050 & 2,49 \\
\hline Usar medicamentos & $-1,03$ & 0,94 & 1,20 & 0,274 & 0,36 & 0,060 & 2,26 \\
\hline Adulto mayor & 0,05 & 0,03 & 3,15 & 0,076 & 1,05 & 0,995 & 1,12 \\
\hline Constante & 1,88 & 2,30 & 0,67 & 0,413 & 6,53 & & \\
\hline
\end{tabular}


La citometría de flujo da una indicación de la medida de inestabilidad genómica determinando la frecuencia de los reticulocitos micronucleados en una población, aunque tiene una única desventaja, y es que no provee información sobre la composición cromosómica de los micronúcleos ni distingue entre los diferentes mecanismos de acción de los inductores (clastogénicos o aneugénicos); el ensayo tampoco determina si una célula tiene un micronúcleo o varios, como sí lo hace la microscopía convencional (6).

Las muestras de sangre de los 25 voluntarios sanos fueron importantes para estimar el número basal de reticulocitos y de aquellos micronucleados. Aunque los porcentajes de estos últimos no fueron tan elevados en este grupo de control $(2,31 \%$; $\mathrm{RIQ}=3,51)$, el coeficiente de variación fue alto $(79,46 \%)$. Al igual que en otros estudios $(1,3,15$ 17), se observó un rango variable de micronúcleos en las frecuencias tanto de los voluntarios sanos como de los pacientes, lo que indica una variabilidad individual significativa. Así, el conocimiento de los cambios espontáneos en las frecuencias de estos eventos permitiría determinar los parámetros basales en la población.

Los análisis de covarianza demostraron que la variación en la frecuencia basal tal vez no está relacionada con la edad y el sexo, lo cual contrasta con otros estudios que sugieren efectos significativos de estos factores en la incidencia de micronúcleos. Tales discrepancias pueden estar relacionadas con diferencias en las células blanco (eritroblastos $V s$. linfocitos) $u$ otros factores inherentes a la variabilidad entre individuos $(3,16)$.

Las frecuencias de micronúcleos antes del tratamiento en los pacientes con cáncer, también fueron variables y más elevadas que las de los voluntarios sanos, con una mediana de $12,45 \%$ ( $R \mid Q=18,32)$, lo que evidenciaría la presencia en sangre circulante de factores genotóxicos de naturaleza aún desconocida, o una posible propensión a padecer la enfermedad, como se ha observado en otros estudios sobre diferentes tipos de cáncer (16-21). Los experimentos aquí descritos sugieren que los reticulocitos ${ }^{\mathrm{CD} 71+}$ micronucleados en sangre periférica podrían representar un posible biomarcador de la propensión a desarrollar este tipo de neoplasias.

Una de las estrategias más prometedoras para la prevención del cáncer es desarrollar y validar biomarcadores que puedan anticiparse al diagnóstico clínico. De hecho, los biomarcadores son instrumentos que ayudan a detectar personas en alto riesgo, y se pueden dividir en tres grupos: el primero, para definir la exposición a agentes carcinogénicos; el segundo, para mostrar efectos biológicos, y el tercero, para dar información sobre la propensión individual $(21,22)$.

El uso de los micronúcleos como biomarcadores de genotoxicidad o riesgo de cáncer (23), se fundamenta en el hecho de que las alteraciones citogenéticas hacen parte del fenotipo tumoral y en la hipótesis de que el daño cromosómico está involucrado directamente en la etiología del cáncer $(21,22)$. Sin embargo, es difícil establecer una relación causal entre el aumento del número de micronúcleos y el riesgo de cáncer, pues dicho aumento puede ser consecuencia de la enfermedad (causalidad inversa) o el reflejo de una propensión individual a eventos que generan inestabilidad genómica $(16,22)$.

La asociación entre las frecuencias elevadas de micronúcleos y el riesgo de desarrollar cáncer, no está confinada a cánceres en sitios específicos (22). Sin embargo, reflejan la acumulación de alteraciones genéticas causadas por factores genotóxicos endógenos y exógenos, así como las variaciones individuales en la vulnerabilidad frente a estos factores $(16,21)$.

Hasta la fecha, las investigaciones sobre biomarcadores en tumores cerebrales han presentado pocas opciones útiles para la práctica clínica, por lo que se hace necesario buscar nuevas opciones de marcadores tumorales, por ejemplo, los basados en sangre periférica o en biopsias líquidas, los cuales resultarían más prácticos que los actuales, ya que se pueden hacer sin necesidad de repetir la cirugía o las biopsias en los pacientes. La investigación de biomarcadores en gliomas de alto grado es todo un reto, dada la baja frecuencia y la diversidad genética de estos tumores. Además, al comparar con otros cánceres, su adopción en la práctica clínica se ha visto obstaculizada por las bajas tasas de detección.

Por último, cabe destacar que la detección de micronúcleos por citometría de flujo en reticulocitos humanos, es una técnica moderna, rápida y eficiente que evalúa la genotoxicidad en los reticulocitos micronucleados con un grado de eficiencia y sensibilidad sin precedentes. Los datos aquí suministrados respaldan la hipótesis de que las frecuencias de estos reticulocitos en sangre periférica pueden ser una alternativa de mayor sensibilidad que los análisis convencionales 
basados en la técnica de linfocitos binucleados, y podrían constituir una nueva herramienta validable como biomarcador para la predicción y la detección temprana del cáncer.

\section{Agradecimientos}

Este estudio recibió el apoyo de la Estrategia de Sostenibilidad 2013/2014 (Grupo GRC) del CODI - Universidad de Antioquia, y la Fundación para la Promoción de la Investigación y la Tecnología del Banco de la República (Proyecto 3640). Los autores agradecen a los donantes voluntarios, y a la Unidad de Quimioterapia del Instituto de Cancerología de la Clínica Las Américas de Medellín, por su ayuda en la obtención de las muestras de los pacientes.

\section{Conflicto de intereses}

Los autores de este artículo expresan que no existieron conflictos de intereses durante la preparación de este documento ni para su publicación.

\section{Financiación}

Esta investigación fue financiada por la Estrategia de Sostenibilidad (Grupo GRC) del CODI Universidad de Antioquia y la Fundación para la Promoción de la Investigación y la Tecnología del Banco de la República (Proyecto 3640).

\section{Referencias}

1. Fenech M, Holland N, Chang WP, Zeiger E, Bonassi S. The Human MicroNucleus Project-An international collaborative study on the use of the micronucleus technique for measuring DNA damage in humans. Mutat Res. 1999;428:271-83. https://doi.org/10.1016/S1383-5742 (99)00053-8

2. Bonassi S, Znaor A, Ceppi M, Lando C, Chang WP, Holland $\mathbf{N}$, et al. An increased micronucleus frequency in peripheral blood lymphocytes predicts the risk of cancer in humans. Carcinogenesis. 2007;28:625-31. https://doi. org/10.1093/carcin/bgl177

3. Dertinger SD, Miller RK, Brewer K, Smudzin T, Torous DK, Roberts DJ, et al. Automated human blood micronucleated reticulocyte measurements for rapid assessment of chromosomal damage. Mutat Res. 2007;626:111-9. https://doi. org/10.1016/j.mrgentox.2006.09.003

4. Araldi RP, de Melo TC, Mendes TB, de Sá Júnior $\mathbf{P}$, Nozima BH, Ito ET, et al. Using the comet and micronucleus assays for genotoxicity studies: A review. Biomed Pharmacother. 2015;72:74-82. https://doi.org/10.1016/j. biopha.2015.04.004

5. Fenech M, Kirsch-Volders M, Natarajan AT, Surralles $\mathrm{J}$, Crott JW, Parry J, et al. Molecular mechanisms of micronucleus, nucleoplasmic bridge and nuclear bud formation in mammalian and human cells. Mutagenesis. 2011;26:125-32. http://dx.doi.org/10.1093/mutage/geq052
6. Balmus G, Karp NA, Ng BL, Jackson SP, Adams DJ, Mclntyre RE. A high-throughput in vivo micronucleus assay for genome instability screening in mice. Nat Protoc. 2014;1:205-15. https://doi.org/10.1038/nprot.2015.010

7. Hanahan D, Weinberg RA. Hallmarks of cancer: The next generation. Cell. 2011;144:646-74. https://doi.org/10.1016/j. cell.2011.02.013

8. von Ledebur $\mathbf{M}$, Schmid $\mathbf{W}$. The micronucleus test. Methodological aspects. Mutat Res. 1973;19:109-17. https://doi.org/10.1016/0027-5107(73)90118-8

9. Schmid W. The micronucleus test. Mutat Res. 1975;31:915. https://doi.org/10.1016/0165-1161(75)90058-8

10. Chen Y, Tsai Y, Nowak I, Wang N, Hyrien O, Wilkins R, et al. Validating high-throughput micronucleus analysis of peripheral reticulocytes for radiation biodosimetry: Benchmark against dicentric and CBMN assays in a mouse model. Health Phys. 2010;98:218-27. https://doi.org/10.1097/ HP.0b013e3181abaae5

11. Dertinger SD, Torous DK, Hayashi M, MacGregor JT. Flow cytometric scoring of micronucleated erythrocytes: An efficient platform for assessing in vivo cytogenetic damage. Mutagenesis. 2011;26:139-45. https://doi.org/10. 1093/mutage/geq055

12. Dertinger SD, Torous DK, Hall NE, Murante FG, Gleason AE, Miller RK, et al. Enumeration of micronucleated CD71-positive human reticulocytes with a single-laser flow cytometer. Mutat Res. 2002;515:3-14. https://doi.org/10. 1016/S1383-5718(02)00009-8

13. Dertinger SD, Chen Y, Miller RK, Brewer K, Smudzin T, Torous DK, et al. Micronucleated CD71-positive reticulocytes: A blood-based endpoint of cytogenetic damage in humans. Mutat Res. 2003;542:77-87. https://doi. org/10.1016/j.mrgentox.2003.08.004

14. Torous DK, Dertinger SD, Hall NE, Tometsko CR. Enumeration of micronucleated reticulocytes in rat peripheral blood: A flow cytometric study. Mutat Res. 2000;465:91-9. https://doi.org/10.1016/S1383-5718(99)00216-8

15. Bonassi S, Fenech M, Lando C, Lin YP, Ceppi M, Chang WP, et al. HUman MicroNucleus project: International database comparison for results with the cytokinesis-block micronucleus assay in human lymphocytes: I. Effect of laboratory protocol, scoring criteria, and host factors on the frequency of micronuclei. Environ Mol Mutagen. 2001;37:3145. https://doi.org/10.1002/1098-2280(2001)37:1<31::AIDEM1004>3.0.CO;2-P

16. Chang P, Li Y, Li D. Micronuclei levels in peripheral blood lymphocytes as a potential biomarker for pancreatic cancer risk. Carcinogenesis. 2010;32:210-5. https://doi. org/10.1093/carcin/bgq247

17. Cao J, Liu Y, Sun H, Cheng G, Pang X, Zhou Z. Chromosomal aberrations, DNA strand breaks and gene mutations in nasopharyngeal cancer patients undergoing radiation therapy. Mutat Res. 2002;504:85-90. https://doi. org/10.1016/S0027-5107(02)00082-9

18. Berg-Drewniok B, Weichenthal M, Ehlert U, Rummelein B, Breitbart EW, Rudiger HW. Increased spontaneous formation of micronuclei in cultured fibroblasts of firstdegree relatives of familial melanoma patients. Cancer Genet Cytogenet. 1997;97:106-10. https://doi.org/10.1016/ S0165-4608(96)00364-0 
19. Scott D, Barber JB, Levine EL, Burrill W, Roberts SA. Radiation-induced micronucleus induction in lymphocytes identifies a high frequency of radiosensitive cases among breast cancer patients: A test for predisposition? $\mathrm{Br} \mathrm{J}$ Cancer. 1998;77:614-20. https://doi.org/10.1038/bjc.1998.98

20. Burril W, Barber JB, Roberts SA, Bulman B, Scott D. Heritability of chromosomal radiosensitivity in breast cancer patients: A pilot study with the lymphocyte micronucleus assay. Int $\mathrm{J}$ Radiat Biol. 2000;76:1617-9. https://doi. org/10.1038/sj.bjc.6600628

21. Bloching M, Hofmann A, Lautenschläger C, Berghaus A, Grummt T. Exfoliative cytology of normal buccal mucosa to predict the relative risk of cancer in the upper aerodigestive tract using the MN-assay. Oral Oncol. 2000;36:550-5. https://doi.org/10.1016/S1368-8375(00)00051-8
22. Murgia E, Ballardin M, Bonassi S, Rossi AM, Barale R. Validation of micronuclei frequency in peripheral blood lymphocytes as early cancer risk biomarker in a nested case-control study. Mutat Res. 2008;639:27-34. https://doi. org/10.1016/j.mrfmmm.2007.10.010

23. Bonassi S, Znaor A, Ceppi M, Lando C, Chang WP, Holland $\mathbf{N}$, et al. An increased micronucleus frequency in peripheral blood lymphocytes predicts the risk of cancer in humans. Carcinogenesis. 2007;28:625-31. https://doi. org/10.1093/carcin/bgl177 